\title{
Adaptation of handwriting size under distorted visual feedback in patients with Parkinson's disease and elderly and young controls
}

\author{
H-L Teulings, J L Contreras-Vidal, G E Stelmach, C H Adler
}

J Neurol Neurosurg Psychiatry 2002;72:315-324

See end of article for authors' affiliations

.....................

Correspondence to: Dr H-L Teulings,

NeuroScript, $1225 \mathrm{E}$

Broadway Road 100

Tempe, AZ 85282, USA;

hlteulings@

neuroscriptsoftware.com

Received

5 December 2000

In revised form 27 July

2001

Accepted 6 August 2001

\begin{abstract}
Objective: The ability to use visual feedback to control handwriting size was compared in patients with Parkinson's disease (PD), elderly people, and young adults to better understand factors playing a part in parkinsonian micrographia.

Methods: The participants wrote sequences of eight cursive I loops with visual target sizes of 0.5 and $2 \mathrm{~cm}$ on a flat panel display digitiser which both recorded and displayed the pen movements. In the pre-exposure and postexposure conditions, the display digitiser showed the actual pen trace in real time and real size. In the distortion exposure conditions, the gain of the vertical dimension of the visual feedback was either reduced to $70 \%$ or enlarged to $140 \%$.

Results: The young controls showed a gradual visuomotor adaptation that compensated for the visual feedback distortions during the exposure conditions. They also showed significant after effects during the postexposure conditions. The elderly controls marginally corrected for the size distortions and showed small after effects. The patients with PD, however, showed no trial by trial adaptations or after effects but instead, a progressive amplification of the distortion effect in each individual trial.

Conclusion: The young controls used visual feedback to update their visuomotor map. The elderly controls seemed to make little use of visual feedback. The patients with Parkinson's disease rely on the visual feedback of previous or of ongoing strokes to programme subsequent strokes. This recursive feedback may play a part in the progressive reductions in handwriting size found in parkinsonian micrographia.
\end{abstract}

$\mathrm{P}$ arkinson's disease (PD) is caused by the degeneration of the dopaminergic nigrostriatal neurons of the basal ganglia ${ }^{1}$ resulting in akinesia, bradykinesia, rigidity, and tremor. Relevant for our study is that patients with Parkinson's disease show deficits in learning new movement patterns, ${ }^{2}$ and deficits of proprioceptive and kinaesthetic feedback ${ }^{3-8}$ In particular, in handwriting movements, patients with Parkinson's disease exhibit more variable peak accelerations and sizes/stroke, ${ }^{9}$ and smaller than normal handwriting — that is, micrographia. ${ }^{10}$ The mechanism of parkinsonian micrographia is not well understood. It has been proposed that it is caused by the reduced kinaesthesia and reduced motor output. ${ }^{3}$ It is unclear why visual feedback does not seem to help patients with Parkinson's disease correct for micrographia whereas they are able to use visual feedback ${ }^{11} 12$ and show normal visual perception during movement. ${ }^{13} 14$ The role of visual feedback in small and fast movements, such as in handwriting, is complex. ${ }^{15}$ Both in the patients with Parkinson's disease and in the elderly controls, the time required to respond to visual feedback changes is at least $160 \mathrm{~ms}^{16}$ which would be within the $200-600 \mathrm{~ms}$ they need to produce a handwriting stroke in this experiment. On the other hand, Denier van der Gon and Thuring ${ }^{17}$ have shown that in fast writers, sudden changes of the visual feedback and proprioceptive feedback may take several handwriting strokes before corrections become manifest.

Fucetola and Smith ${ }^{18}$ have tested the effects of display gain distortions in patients with Parkinson's disease and elderly people. They used a digitiser while the visual feedback was presented on a separate computer display positioned in front of the participant so that a visuomotor transformation was required between the display space and the writing space. Their results showed that both the patients with Parkinson's disease and elderly people corrected equally well for the distortions. In the present study we used a novel technique where participants wrote on a display digitiser (a digitiser overlaying a flat panel liquid crystal display) which allowed us to manipulate the visual size of the handwriting trace while the participants monitored both their hand and their pen traces. To examine whether visual feedback is used to control handwriting size we introduced size distortions of the visual feedback of the pen trace and examined the effects on the actual handwriting movement size.

\section{PATIENTS AND METHODS}

\section{Subjects}

Eleven patients with Parkinson's disease (nine men and two women; age range 51-79; mean (SD) 70.9 (7.7)), 16 elderly controls (six men and 10 women; age range 64-81; mean (SD) $71.5(5.2)$ ), and 10 young controls (seven men and three women; age range 22-31; mean (SD) 25.9 (2.5)) participated in the experiment after informed consent. One additionally tested patient with Parkinson's disease was excluded because his data yielded many segmentation errors in the data analysis. The patients and the elderly group received a financial reward for participating. All participants were right handed except one young and one elderly control who were left handed. We assumed that handedness did not play a part. All participants had normal or corrected to normal visual acuity. The patients were diagnosed with Parkinson's disease 1 to 16

Abbreviations: PD, Parkinson's disease; LCD, liquid crystal display SEM, standard error of the mean. 


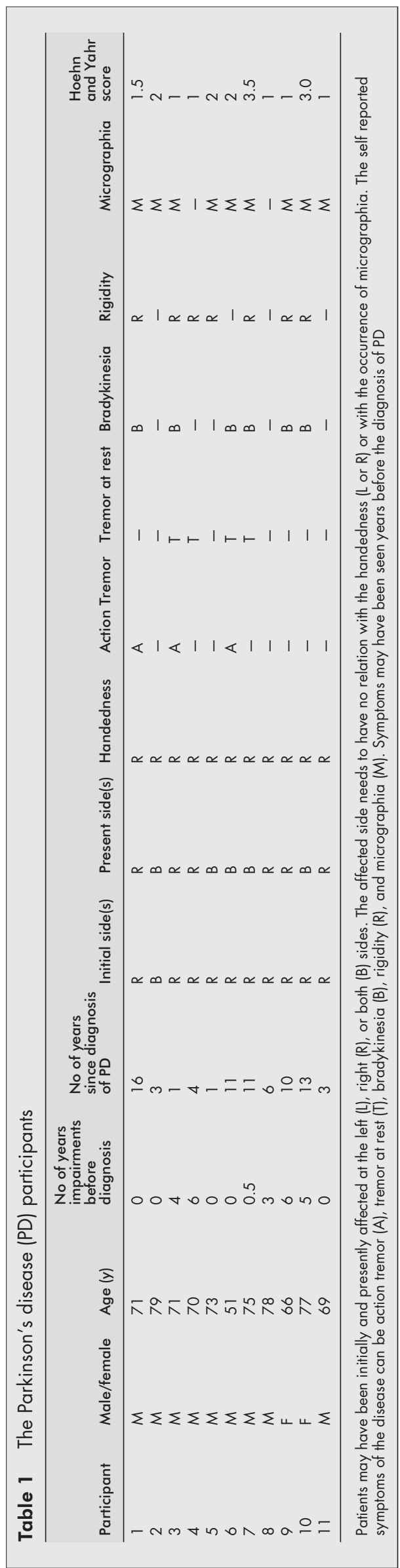

years earlier (mean 7.2 (SD 5.2)) and had Hoehn and Yahr scores ranging from 1 to 3.5 (mean 1.7 (SD 0.9) (table 1). The patients were asked to voluntarily skip their morning dose of antiparkinsonian medication if they felt comfortable and were tested in the morning. Those who felt comfortable to skip their morning dose were the more mild patients and were tested during their "off" state, whereas the other patients were tested during their "on" state. Nine out of the 11 patients reported that they sometimes had micrographic writing impairments-for example, when writing for extensive periods while not paying attention to their writing or with increased mental load. ${ }^{19}$ The patients with Parkinson's disease were either right sided or bilaterally affected. Micrographia is a central phenomenon, unrelated to handedness or the affected side. ${ }^{10}$

\section{Apparatus}

The participants wrote on a display digitiser (Wacom pl-100v) controlled by a PC. The display digitiser is a digitiser overlaying a flat panel display. It rested on top of a table and could be shifted and rotated to meet the participant's preferred orientation. The display of the digitiser was a $640 \times 480$ points (VGA), 16 grey level, back lit, liquid crystal display (dot pitch $0.03 \mathrm{~cm}$ which was equivalent to a display root mean squared error of $0.01 \mathrm{~cm}$ ). The room illumination was slightly dimmed to allow optimal vision of the back lit display. The display digitiser had an active area of $19.5 \mathrm{~cm}$ wide and $14.5 \mathrm{~cm}$ deep at 6 $\mathrm{cm}$ from its front side. The writing window was $18 \mathrm{~cm}$ wide and $10 \mathrm{~cm}$ deep and was centred on the active area of the digitiser. The writing window showed a horizontal baseline $6 \mathrm{~cm}$ from the bottom of the writing window. The digitiser sampled the $x$ and $y$ coordinates of the pen tip at a rate of 200 times/second (resolution $0.005 \mathrm{~cm}$, root mean squared error $0.01 \mathrm{~cm})$. The pen pressure was recorded to detect whether the pen touched the digitiser so that a continuous line had to be displayed. Recording started as soon as the pen touched the surface of the display digitiser and ended when the pen was lifted for more than 0.5 seconds. A low pitch beep signalled that the digitiser was ready for recording. A high pitch beep sounded when the recording period was timed out. Within seconds, the system was ready to record again.

\section{Tasks and instructions}

The participants were made familiar with the display digitiser by writing their name and a test sentence twice each. The test sentence was repeated twice at the end of the experiment to evaluate effects of fatigue or medication during the 60 minute experimental session. We found no differences between first and last test sentences. The participants were instructed to perform a sequence of about eight cursive loops (fig l, left hand panels) but were to refrain from counting the loops as the recording system showed the number of loops immediately after each trial. If the number of loops was less than six, the trial was redone.

The participants performed the writing pattern in two blocks in random order with target sizes $0.5 \mathrm{~cm}$ and $2 \mathrm{~cm}$, respectively. The blocked presentation of trials minimises task switching, which would disadvantage the patients with Parkinson's disease ${ }^{20}$ Each block started with a training condition where the target size was indicated by two parallel, horizontal guidelines. During the subsequent experimental conditions, only the baseline was shown and the target size was indicated by a small horizontal marker on the left margin of the display digitiser. This target size indicator was intentionally degraded because normal guidelines ${ }^{10}$ or target dots $^{21}$ would enable even the patients with Parkinson's disease to produce the target sizes with little error.

The visual feedback was presented via the display digitiser, either real size $(100 \%)$ or distorted by changing the vertical display gain to $70 \%$ or $140 \%$. Only the vertical dimension was 


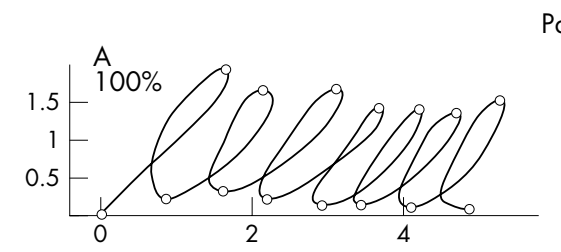

Parkinsonian
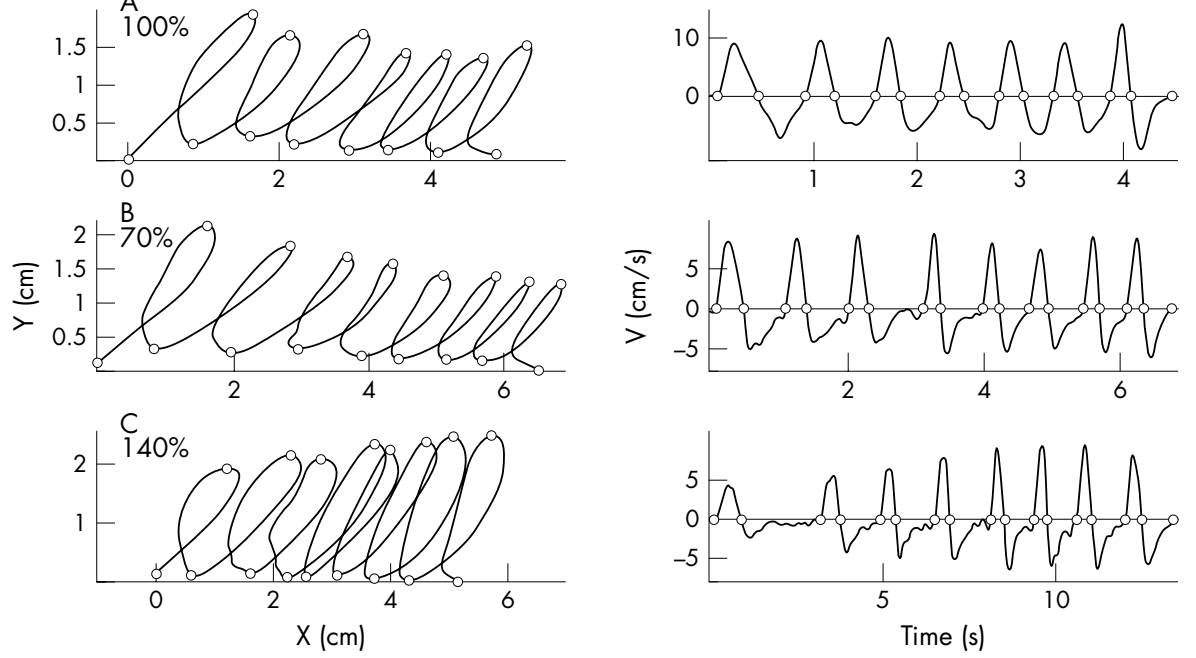

Figure 1 Loop patterns with visible target size of $2 \mathrm{~cm}$ produced by a patient with Parkinson's disease under the normal $100 \%$ visual feedback condition (A, left panel), and under two distorted visual feedback conditions where the vertical gain of the display is reduced to $70 \%$ (B), or enlarged to $140 \%$ (C). The vertical velocity as a function of time is shown in the right panels. Unlike the controls, who learn trial by trial to correct for the distortion, the patients with Parkinson's disease progressively amplify the effects of the distortions during each trial again without evidence of learning. The patients also adapted the horizontal size but in a corrective way, similar to the controls. Consequently, in this experiment where only the vertical dimension was distorted, the patient's handwriting becomes progressively smaller but wider in the $70 \%$ condition (B) and progressively larger but narrower in the 140\% condition (C).

rescaled because rescaling the horizontal dimension would result in a too obvious, growing discrepancy between the current pen position and the distorted trace while progressing from left to right. Similarly, to prevent a visible offset of the distorted writing trace, in case the participant writes above or below the baseline, the distortion left the start position of each upward stroke intact. Thus, the participants could not readily discover the nature of the visual feedback distortion. The participants were instructed to write the l pattern such that vertical size of the visible patterns matched the target size. To achieve the target size in the $70 \%$ visual feedback condition, they needed to enlarge their stroke sizes to $100 / 70=143 \%$ and in the $140 \%$ condition they needed to reduce their stroke sizes to $100 / 140=71 \%$.

Within each target size block, the gain of the vertical dimension of the visual feedback was varied in a fixed sequence of six trial blocks to minimise the variability between participants. The sequence of feedback conditions for each target size was:

(1) Training condition with vertical gain of $100 \%$, with both base and top lines to learn the target sizes.

(2) Pre-exposure condition with vertical gain of $100 \%$, with only a baseline and a small size marker in the left margin.

(3) First exposure condition with vertical gain of $70 \%$ to measure adaptation to the distortion.

(4) First postexposure condition with vertical gain of 100\% to measure the after effects to verify that adaptation took place.

(5) Second exposure condition with vertical gain of $140 \%$ to measure adaptation to the distortion.

(6) Second postexposure condition with vertical gain of $100 \%$ to measure after effects.

The experimental data consisted of conditions 2 to 6 . To specifically evaluate whether the participants performed the correct target size in the pre-exposure condition we compared conditions $\mathrm{l}$ and 2. To evaluate the adaptation to the distortion, the first $100 \%$ (pre-exposure) condition was compared with the $70 \%$ and $140 \%$ exposure conditions (conditions 2, 3, and 5, respectively). To evaluate the after effects, ${ }^{22}{ }^{23}$ the pre-exposure condition was compared with the two postexposure conditions that followed the $70 \%$ and $140 \%$ distortion conditions (conditions 2, 4, and 6, respectively).

\section{Movement feature extraction and analysis}

The handwriting recordings were filtered using a frequency domain low pass filter at $7 \mathrm{~Hz}$ (pass band 0 to $3 \mathrm{~Hz}$, cosine transition band between 3 and $11 \mathrm{~Hz}$, complete suppression above $11 \mathrm{~Hz}) .^{24}$ The beginning and end points of the writing pattern were first roughly located by the first and the last sample, respectively, where the absolute vertical velocity exceeded $5 \%$ of the absolute peak velocity. Subsequently, the beginning and end points of the patterns were located more precisely by searching in the opposite direction for respectively the first and last zero crossing or reversal. Finally, the segmentation points were estimated by linear interpolation between the two successive samples where the vertical velocity crosses the zero level. Each stroke pattern was thus segmented into alternating up and down strokes and occasional segmentation points between two consecutive up strokes or two consecutive down strokes were removed. A stroke pattern verifier was used to check that each pattern consisted of a sequence of counterclockwise loops. Initial downward strokes were removed. Any trials with less than six up-down stroke pairs, or that did not form six cursive l loops, were discarded. The proportion of discarded trials was $2.5 \%$ in the young, $4.2 \%$ in the elderly controls, and $13.4 \%$ in the patients with Parkinson's disease.

We calculated vertical stroke size by the absolute difference in vertical position between the beginning and end of each stroke. The absolute vertical stroke size, averaged across an up and down stroke pair provides information on how the participants responded to the distortions. Similarly, the horizontal stroke size was calculated by the absolute difference in horizontal positions. Both horizontal and vertical sizes were estimated because the motor system is able to control these measures independently. ${ }^{25}$ Stroke duration was estimated by the difference in time between successive segmentation points. Increases of durations in the distortion conditions may provide information whether additional processing takes place to perform the transformations. ${ }^{26}$ Horizontal stroke size and stroke duration are only reported where relevant. Stroke duration showed only small effects, but is reported to allow comparison with other studies. The data were non-gaussian, as outliers generally overshot the average. We therefore used the conservative Wilks' $\lambda$ correction of the $F$ values of the analysis of variance (ANOVA). 
Target size $0.5 \mathrm{~cm}$
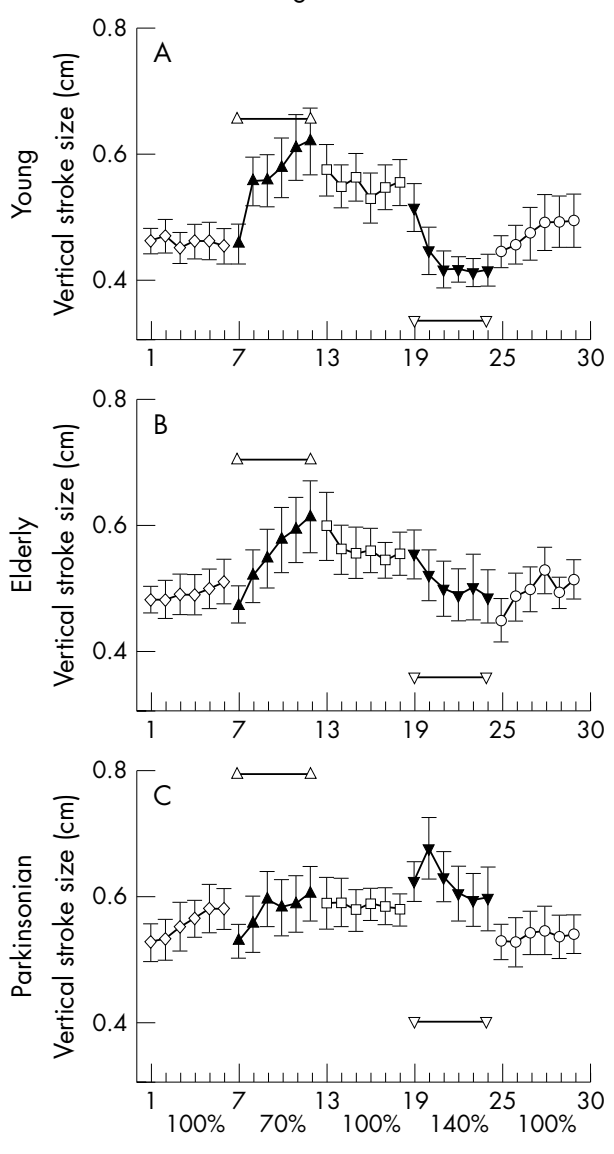

Trials
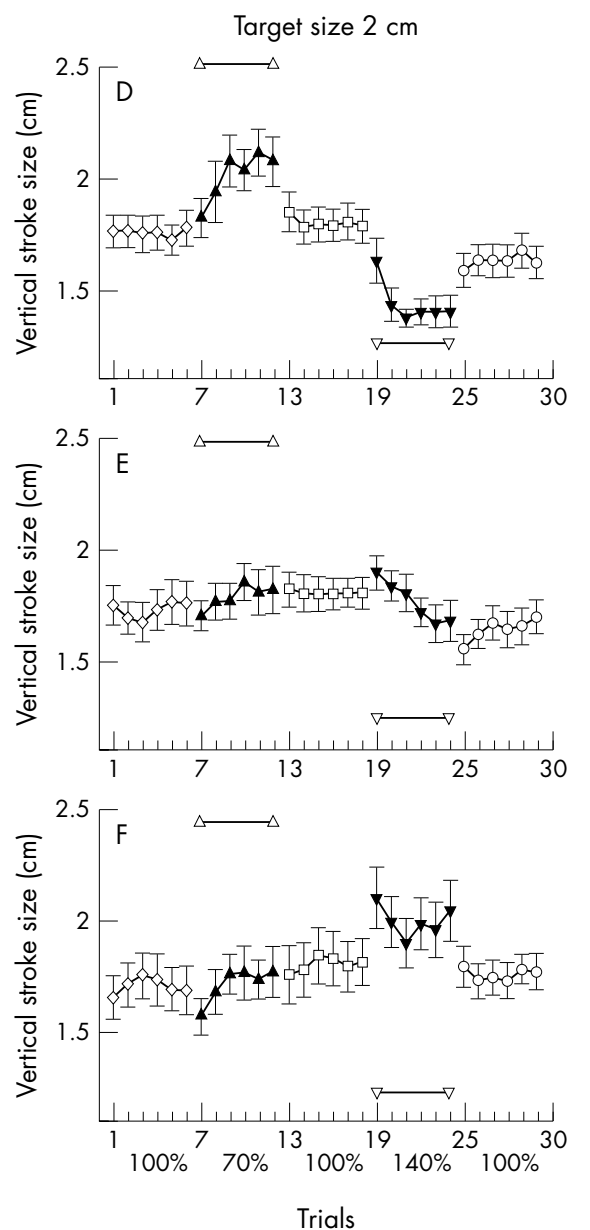

Figure 2 Chronological sequence of the trial averages of vertical stroke size for the $0.5 \mathrm{~cm}$ target size in young controls (A), elderly controls (B), and patients with Parkinson's disease (C) and similarly for the $2 \mathrm{~cm}$ target size (D, E, F). Vertical bars are standard error of the mean (SEM). The sequence of the vertical gain distortions of the visual feedback was 100\% (real size, pre-exposure condition in trials 1-6), 70\% (reduced in trials 7-12), 100\% (first post-exposure in trials 13-18), 140\% (enlarged in trials 19-24), and 100\% (second postexposure in trials 25-30). The initial six training trials are not shown. Based on the actual pre-exposure loop sizes, the perfect size corrections in the 70\% and the $140 \%$ conditions are shown by the horizontal line segments. The young controls learned trial by trial to adapt the ideal correction whereas the patients with Parkinson's disease did not show learning or correction but showed amplification of the effects of the distortions. The elderly patients showed responses between the young controls and the patients with Parkinson's disease.

To verify whether our experiment could have too small statistical power, we present effect size and statistical power for effects that failed to reach significance. The effect size, $\eta^{2}$, estimates the proportion of the total variability of the data that can be accounted for by the experimental factors. As a rule of thumb, effect sizes between 0 and 0.06 signify nil or small effects and effect sizes between 0.15 and 1 signify strong effects. The statistical power (assuming $\mathrm{p}=0.05$ ) is the probability of correctly accepting that there is an effect. As a rule of thumb, a power between 0 and 0.2 suggests a low power test and a power between 0.8 and 1 suggests a test of high power. Furthermore, the variances were not homogeneous as the Huynh-Feldt's $\epsilon$ of sphericity ranged between 0.30 and 0.75 . Therefore, in addition to the post hoc parametric tests-for example, Student's $t$ tests for unpaired or paired data, we also reported any significant non-parametric statistical tests-for example, the MannWhitney-Wilcoxon $U$ test for between group comparisons (unpaired data) or the sign test for within group comparisons (paired data, assuming a $50 \%$ a priori chance). ${ }^{27} 28$

\section{RESULTS}

Figure 2 represents the course of the vertical stroke size during the sequence of conditions and trials per group and target size. The average stroke features (vertical and horizontal size, and duration) per group, target size, and feedback condition have been summarised in table 2 . Where possible, we collapsed the data across the six loops and the up and down strokes to reduce the complexity of the ANOVA. The results were analyzed as a function of the five relevant factors: group, target size condition, feedback condition (in various comparisons), trial, and loop. Differences between up and down strokes did not show significant effects and are omitted.

\section{Absence of group main effects}

Firstly, we verified that the three groups were sufficiently comparable so that any multiplicative effects would not cause group interactions (table 2). A three groups $\times$ two sizes $\times$ five conditions $\times$ six loops ANOVA (SPSS general linear models for repeated measures) of the vertical stroke size/loop confirmed that the groups were not significantly different $(F(2,34)=0.5$, $\mathrm{p}>0.5, \eta^{2}=0.03$, power $=0.13$ ). In this analysis, all conditions, except the initial training condition, were included. Although nine of the 11 patients with Parkinson's disease reported that they sometimes had micrographia (table 1), they did not show signs of micrographia in this experiment because we used clear target sizes. By contrast, their horizontal stroke sizes marginally differed between the groups $(F(2,34)=2.6, \mathrm{p}=0.08)$. The difference was most obvious between the young and the elderly groups $(F(1,24)=51, \mathrm{p}<0.01)$ and was caused by the different handwriting styles and slants between the younger and the 


\begin{tabular}{|c|c|c|c|c|c|c|c|c|}
\hline \multirow[b]{3}{*}{ Subject group } & \multirow{3}{*}{$\begin{array}{l}\text { Target size } \\
(\mathrm{cm})\end{array}$} & \multirow{3}{*}{$\begin{array}{l}\text { Visual feedback distortion } \\
\text { condition }\end{array}$} & \multicolumn{4}{|l|}{ Stroke size } & \multirow{2}{*}{\multicolumn{2}{|c|}{ Stroke duration }} \\
\hline & & & \multicolumn{2}{|l|}{ Vertical } & \multicolumn{2}{|l|}{ Horizontal } & & \\
\hline & & & Mean $(\mathrm{cm})$ & $\mathrm{SD}(\mathrm{cm})$ & Mean $(\mathrm{cm})$ & $\mathrm{SD}(\mathrm{cm})$ & Mean $(\mathrm{cm})$ & $\mathrm{SD}(\mathrm{cm})$ \\
\hline \multirow{12}{*}{$\begin{array}{l}\text { Young controls } \\
(n=10)\end{array}$} & 0.5 & $100 \%$ (training) & 0.465 & 0.061 & 0.273 & 0.086 & 0.220 & 0.065 \\
\hline & 0.5 & $100 \%$ (baseline) & 0.457 & 0.074 & 0.266 & 0.059 & 0.196 & 0.046 \\
\hline & 0.5 & $70 \%$ & 0.561 & 0.129 & 0.305 & 0.070 & 0.208 & 0.058 \\
\hline & 0.5 & $100 \%$ (after $70 \%$ ) & 0.549 & 0.109 & 0.279 & 0.068 & 0.186 & 0.046 \\
\hline & 0.5 & $140 \%$ & 0.431 & 0.078 & 0.227 & 0.057 & 0.200 & 0.065 \\
\hline & 0.5 & $100 \%$ (after $140 \%$ ) & 0.469 & 0.110 & 0.254 & 0.049 & 0.182 & 0.042 \\
\hline & 2 & $100 \%$ (training) & 1.816 & 0.107 & 0.719 & 0.378 & 0.317 & 0.097 \\
\hline & 2 & $100 \%$ (baseline) & 1.754 & 0.218 & 0.636 & 0.352 & 0.273 & 0.092 \\
\hline & 2 & $70 \%$ & 2.006 & 0.308 & 0.754 & 0.376 & 0.295 & 0.101 \\
\hline & 2 & $100 \%$ (after $70 \%$ ) & 1.795 & 0.225 & 0.673 & 0.342 & 0.267 & 0.081 \\
\hline & 2 & $140 \%$ & 1.431 & 0.181 & 0.573 & 0.221 & 0.285 & 0.090 \\
\hline & 2 & $100 \%$ (after $140 \%$ ) & 1.619 & 0.213 & 0.678 & 0.267 & 0.253 & 0.066 \\
\hline \multirow{12}{*}{$\begin{array}{l}\text { Elderly controls } \\
(n=16)\end{array}$} & 0.5 & $100 \%$ (training) & 0.481 & 0.077 & 0.374 & 0.113 & 0.269 & 0.153 \\
\hline & 0.5 & $100 \%$ (baseline) & 0.490 & 0.110 & 0.364 & 0.108 & 0.277 & 0.181 \\
\hline & 0.5 & $70 \%$ & 0.550 & 0.175 & 0.432 & 0.150 & 0.298 & 0.222 \\
\hline & 0.5 & $100 \%$ (after $70 \%$ ) & 0.558 & 0.136 & 0.434 & 0.160 & 0.275 & 0.169 \\
\hline & 0.5 & $140 \%$ & 0.502 & 0.160 & 0.362 & 0.138 & 0.279 & 0.159 \\
\hline & 0.5 & $100 \%$ (after $140 \%$ ) & 0.488 & 0.119 & 0.376 & 0.124 & 0.287 & 0.192 \\
\hline & 2 & $100 \%$ (training) & 1.712 & 0.161 & 1.100 & 0.467 & 0.354 & 0.217 \\
\hline & 2 & $100 \%$ (baseline) & 1.728 & 0.330 & 1.124 & 0.532 & 0.323 & 0.197 \\
\hline & 2 & $70 \%$ & 1.784 & 0.284 & 1.098 & 0.414 & 0.353 & 0.230 \\
\hline & 2 & $100 \%$ (after $70 \%$ ) & 1.801 & 0.260 & 1.073 & 0.464 & 0.336 & 0.216 \\
\hline & 2 & $140 \%$ & 1.759 & 0.236 & 0.962 & 0.365 & 0.342 & 0.205 \\
\hline & 2 & $100 \%$ (after $140 \%$ ) & 1.629 & 0.258 & 1.007 & 0.407 & 0.333 & 0.214 \\
\hline \multirow{12}{*}{$\begin{array}{l}\text { Patients with PD } \\
(n=11)\end{array}$} & 0.5 & $100 \%$ (training) & 0.504 & 0.072 & 0.435 & 0.276 & 0.418 & 0.374 \\
\hline & 0.5 & $100 \%$ (baseline) & 0.555 & 0.099 & 0.481 & 0.310 & 0.460 & 0.483 \\
\hline & 0.5 & $70 \%$ & 0.580 & 0.136 & 0.531 & 0.305 & 0.535 & 0.686 \\
\hline & 0.5 & $100 \%$ (after $70 \%$ ) & 0.582 & 0.100 & 0.517 & 0.295 & 0.426 & 0.360 \\
\hline & 0.5 & $140 \%$ & 0.594 & 0.102 & 0.492 & 0.302 & 0.489 & 0.444 \\
\hline & 0.5 & $100 \%$ (after $140 \%$ ) & 0.525 & 0.109 & 0.485 & 0.293 & 0.457 & 0.434 \\
\hline & 2 & $100 \%$ (training) & 1.644 & 0.253 & 1.092 & 0.719 & 0.672 & 0.658 \\
\hline & 2 & $100 \%$ (baseline) & 1.716 & 0.340 & 1.135 & 0.823 & 0.653 & 0.687 \\
\hline & 2 & $70 \%$ & 1.704 & 0.278 & 1.116 & 0.813 & 0.660 & 0.642 \\
\hline & 2 & $100 \%$ (after $70 \%$ ) & 1.781 & 0.385 & 1.142 & 0.834 & 0.684 & 0.685 \\
\hline & 2 & $140 \%$ & 1.936 & 0.347 & 0.979 & 0.537 & 0.997 & 1.160 \\
\hline & 2 & $100 \%$ (after $140 \%$ ) & 1.712 & 0.199 & 1.000 & 0.616 & 0.736 & 0.760 \\
\hline
\end{tabular}

elderly generations. Handwriting slant was estimated by the direction of the least squares line fitted through the down strokes. ${ }^{29}$ The young controls wrote upright (mean (SD) of the slant measured counterclockwise from the baseline direction was 87 (6) degrees) compared with the slanted writing style of the elderly controls (67 (10) degrees) and the patients (67 (18) degrees). We have no reason to assume that this difference in style or slant causes any of the other group effects or interactions reported here. Finally, stroke duration in the young controls, the elderly controls, and the patients seemed different $(0.235 \mathrm{~s}, 0.311 \mathrm{~s}$, and $0.609 \mathrm{~s}$, respectively (table 2$))$, but this difference was marginal $(F(2,34)=3.2, \mathrm{p}=0.05)$. We concluded that no group differences existed that could introduce major group interactions.

\section{Differences between the $\mathbf{0 . 5}$ and $\mathbf{2} \mathrm{cm}$ target sizes}

When comparing the two target sizes of $0.5 \mathrm{~cm}$ and $2 \mathrm{~cm}$, the large dynamic range caused inhomogeneous variances because standard deviations generally varied proportionally with averages (fig 2 and table 2). Furthermore, uninteresting, multiplicative size effects may show as highly significant interactions. To turn these multiplicative effects into additive (non-interacting) differences, all data for this target-size analysis were log transformed and entered in three groups $\times$ two sizes $\times$ three conditions $\times$ six loops ANOVA, comparing only the pre-exposure and the two postexposure conditions. The significant target size effects on the vertical stroke size were trivial. More importantly, target size did not show significant interactions with any of the other factors. In particular, no group by target size interaction was found $\left(F(2,34)=1.2, \mathrm{p}>0.2, \eta^{2}=0.069\right.$, power $\left.=0.25\right)$. Interestingly, the average vertical stroke size in the $2 \mathrm{~cm}$ target size was $1.72 \mathrm{~cm}$. Apparently, the large target size was undershot in all groups (the 95\% confidence intervals (95\% CIs) for each undistorted feedback condition and for each group were less than $1.98 \mathrm{~cm}$ ). By contrast, each group produced the $0.5 \mathrm{~cm}$ target size well within the $95 \%$ CIs in the undistorted feedback conditions. Similarly, the horizontal stroke size differed between the 0.5 and the $2 \mathrm{~cm}$ target sizes, but no significant group by size interactions were found $\left(F(2,33)=2.6, \mathrm{p}>0.05, \eta^{2}=0.14\right.$, power $=0.49$ ). Finally, stroke duration differed between the 0.5 and the $2 \mathrm{~cm}$ target sizes: 0.306 seconds versus 0.430 seconds, respectively $(F(1,34)=54, \mathrm{p}<.001)$, but again group by size interaction was non-significant $\left(F(2,34)=2.5, \mathrm{p}>0.05, \eta^{2}=0.13\right.$, power $=0.47$ ).

\section{Training versus pre-exposure conditions}

We verified that the target sizes learned during the training conditions (where both the baseline and top line as size 

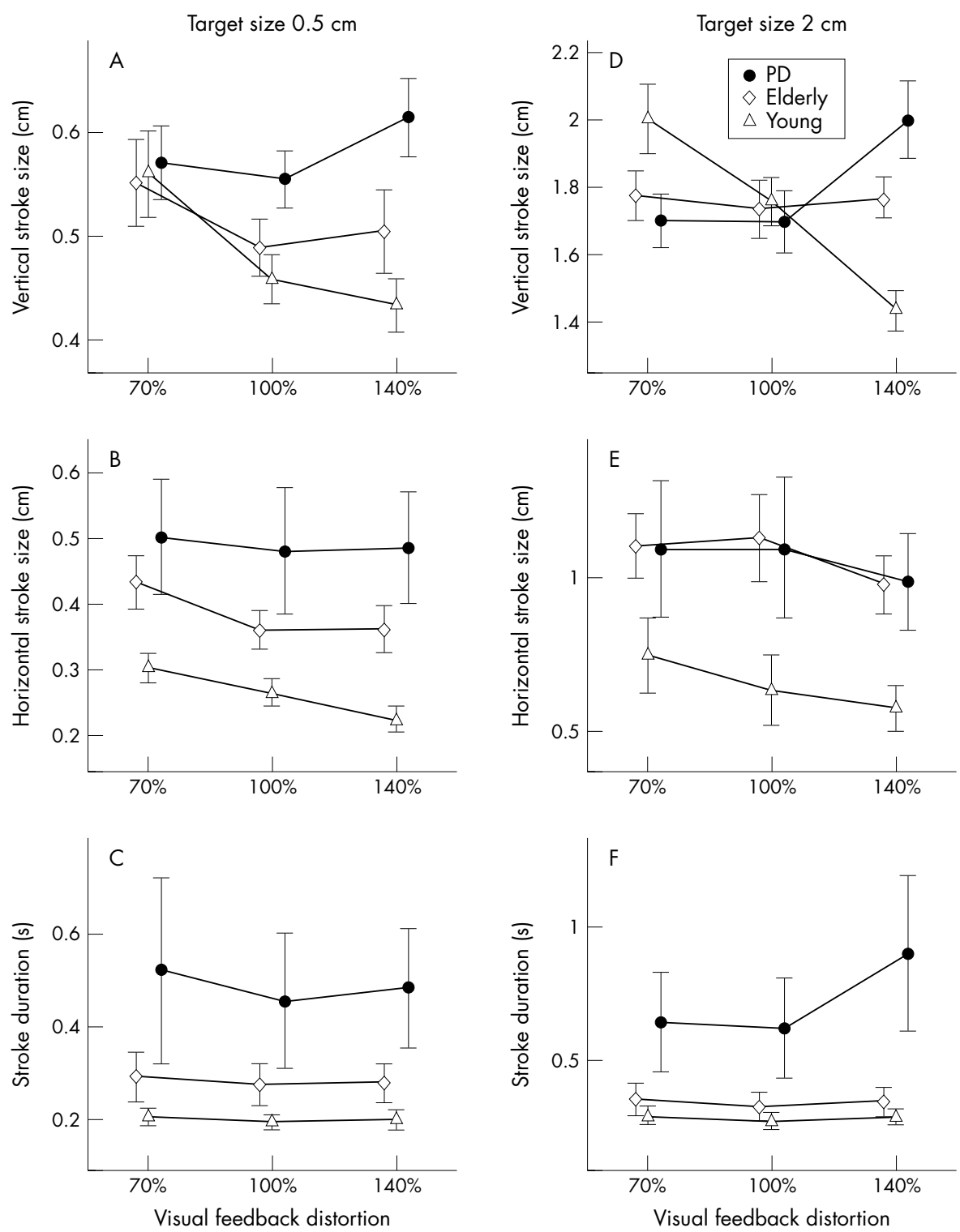

Figure 3 Average vertical sizes for the 100\% pre-exposure and the $70 \%$ and the $140 \%$ vertical gain distorted visual feedback conditions in the $0.5 \mathrm{~cm}(A)$ and the $2 \mathrm{~cm}$ target sizes $(D)$ in the young controls, the elderly controls, and the patients with Parkinson's disease, and, similarly, the average horizontal sizes (B, E) and the average stroke durations (C, F). Vertical bars are SEM. Whereas the young controls show the expected correction of both horizontal and vertical stroke sizes, the patients with Parkinson's disease show, in the 140\% condition, amplification of the effects of the visual feedback distortion but the normal adaptation of the horizontal stroke size.

indicator were presented), were correctly produced during the pre-exposure condition (where the top line was replaced by a small horizontal marker in the left margin). Vertical stroke size was not affected by the transition from the training condition to the pre-exposure condition as supported in a three groups $\times$ two target sizes $\times$ two conditions $\times$ six loops ANOVA that included only the training and the pre-exposure conditions. Both the condition effect $(F(1,34)=0.46, \mathrm{p}>0.5$, $\eta^{2}=0.013$, power $\left.=0.10\right)$ and the condition by group interaction $\left(F(2,34)=1.31, \mathrm{p}>0.2, \eta^{2}=0.072\right.$, power $\left.=0.26\right)$ were nonsignificant.

\section{Distortion effects: pre-exposure versus distortion conditions}

The young controls corrected their vertical stroke size by increasing it in the $70 \%$ condition, in both the $0.5 \mathrm{~cm}$ and the 2 $\mathrm{cm}$ target sizes $(t(9)=4.1, \mathrm{p}<0.01$; sign test, $\mathrm{n}=10, k=0$, $\mathrm{p}<0.01$, and $\mathrm{t}(9)=4.9, \mathrm{p}<0.001$; sign test, $\mathrm{n}=10, k=0, \mathrm{p}<0.01$, respectively). Conversely, in the $140 \%$ condition, they decreased vertical stroke size, which was significant only in the $2 \mathrm{~cm}$ target sizes $(t(9)=4.3, \mathrm{p}<0.01)$. Although the young controls showed the appropriate corrections of the vertical stroke size during the feedback distortion conditions, it is remarkable that the patients with Parkinson's disease showed the opposite tendency as they amplify the effect of the visual feedback distortions (fig 2) - for example, they increased vertical stroke size in the $140 \%$ condition where the vertical strokes size was already enlarged. This is supported by a group by condition interaction $(F(4,66)=5.5, \mathrm{p}<0.001)$ in a three groups $\times$ two target sizes $\times$ two conditions $\times$ six loops ANOVA comparing only the pre-exposure and the two distortion exposure (70\% and 140\%) conditions. The elderly controls showed results in between those of the young controls and the patients with Parkinson's disease.

Although the visual feedback distortion did not affect the horizontal dimension of the visual feedback, all groups changed their horizontal stroke sizes as supported by a significant condition effect $(F(2,33)=9.8, \mathrm{p}<0.001)$. The adaptation was similar in all groups as we did not find a group 

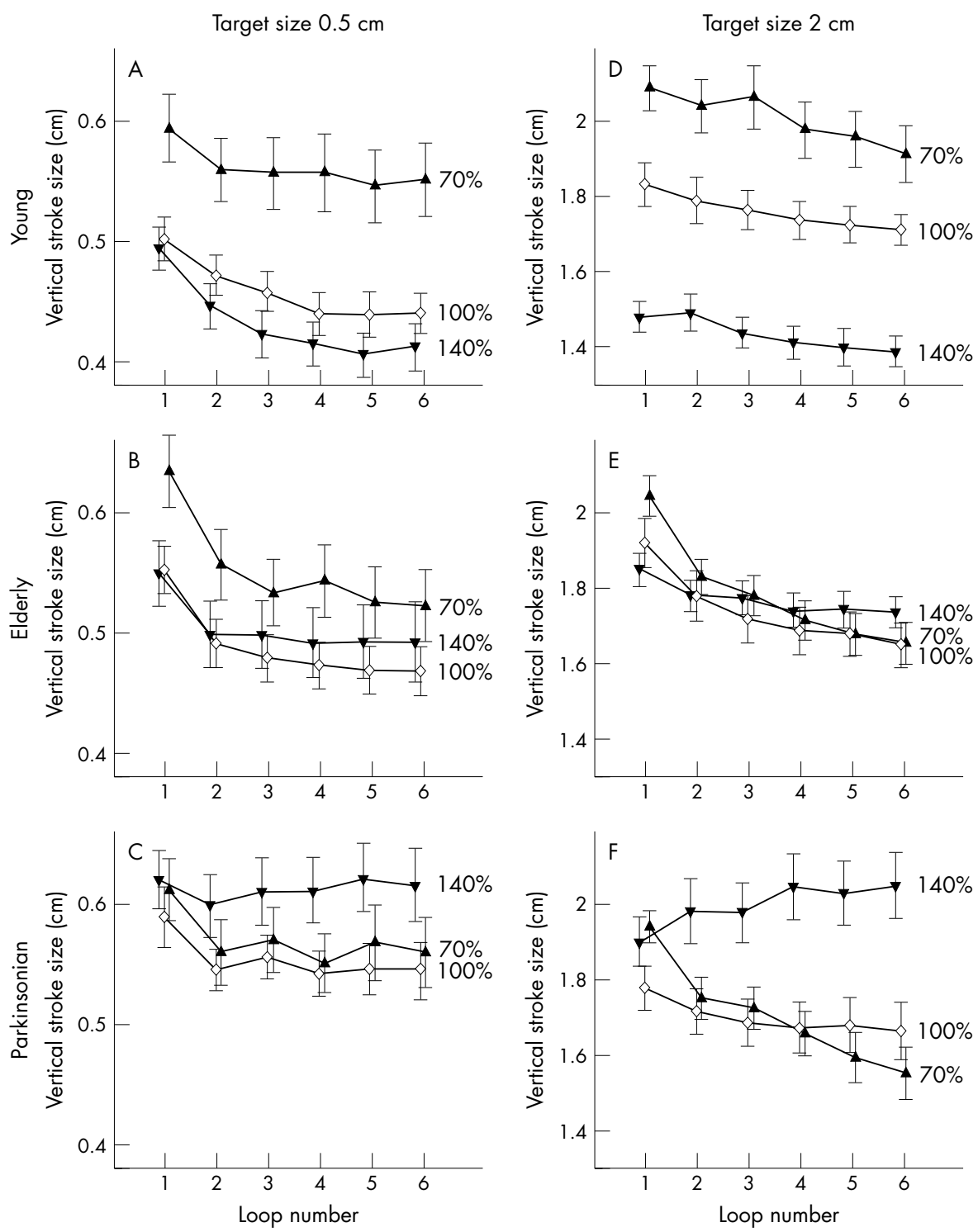

Figure 4 Vertical stroke size as a function of loop number for $(A)$ the $0.5 \mathrm{~cm}$ target size in the young controls, $(\mathrm{B})$ the elderly controls, and (C) the patients with Parkinson's disease for the 70\%, the 100\%, and the 140\% visual feedback conditions and similarly for the $2 \mathrm{~cm}$ target size $(D, E, F)$. The vertical bars are SEM. In the $2 \mathrm{~cm}$ target size, the patients with Parkinson's disease show, in the $70 \%$ condition, a trend of progressive reduction, and, in the $140 \%$ condition, a trend of progressive enlargement.

by condition interaction $\left(F(4,66)=0.81, \mathrm{p}>0.5, \eta^{2}=0.046\right.$, power $=0.25$ ). The young controls increased horizontal sizes in the $70 \%$ feedback condition (fig $3 \mathrm{~B}$ and $\mathrm{E}$ ) in a similar fashion as the vertical stroke size (fig $3 \mathrm{~A}$ and $\mathrm{D}$ ), and vice versa in the $140 \%$ condition. This suggests that the young controls maintained the "movement aspect ratio" between horizontal and vertical movement amplitudes. In the $70 \%$ condition, also the patients with Parkinson's disease increased the horizontal size but, as shown before, decreased the vertical size, resulting in smaller but stretched loops (compare fig $\mathrm{l}$ A and B). Conversely, in the $140 \%$ condition, the patients produced larger but narrower loops (compare fig $\mathrm{l} \mathrm{A}$ and $\mathrm{C}$ ). This effect occurred most clearly in the $2 \mathrm{~cm}$ target sizes. The elderly controls showed results in between those of the young controls and the patients.

\section{After effects: pre-exposure versus postexposure conditions}

After effects of vertical stroke size in the postexposure conditions $(100 \%)$ after the $70 \%$ and $140 \%$ distortion conditions allowed us to verify whether the participants learned to anticipate a correction for the distortion, which subsequently had to be unlearned (fig 2). Small but significant after effects were found using a three groups $\times$ two target sizes $\times$ three conditions $\times$ six loops ANOVA $(F(2,33)=11.5, p<0.001)$ when comparing the initial pre-exposure condition and the two postexposure conditions (after the $70 \%$ and $140 \%$ visual feedback conditions). The after effects following the $70 \%$ and $140 \%$ conditions have, of course, opposite directions as supported by a quadratic contrast $(F(1,34)=15, \mathrm{p}<0.001)$ without a linear trend $(F(1,34)=3.4, \mathrm{p}=0.07)$. When analyzing the after effects for each group separately, however, we found that they were significant in the young $(F(2,8)=22$, $\mathrm{p}<0.001$ ) (fig $2 \mathrm{~A}$ and $\mathrm{D}$ ) and the elderly controls $(F(2,14)=1 \mathrm{l}, \mathrm{p}<0.001)$ (fig $2 \mathrm{~B}$ and $\mathrm{E})$, but not in the patients $\left(F(2,9)=1.02, \quad \mathrm{p}>0.2, \quad \eta^{2}=0.19, \quad\right.$ power $\left.=0.18\right) \quad($ fig $2 \mathrm{C}$ and $F$ ).

\section{Trial by trial adaptation}

In this analysis, missing trials were recursively substituted by the next trial or, if no following trial existed, the previous trial. This substitution makes the learning curves flatter and cannot 

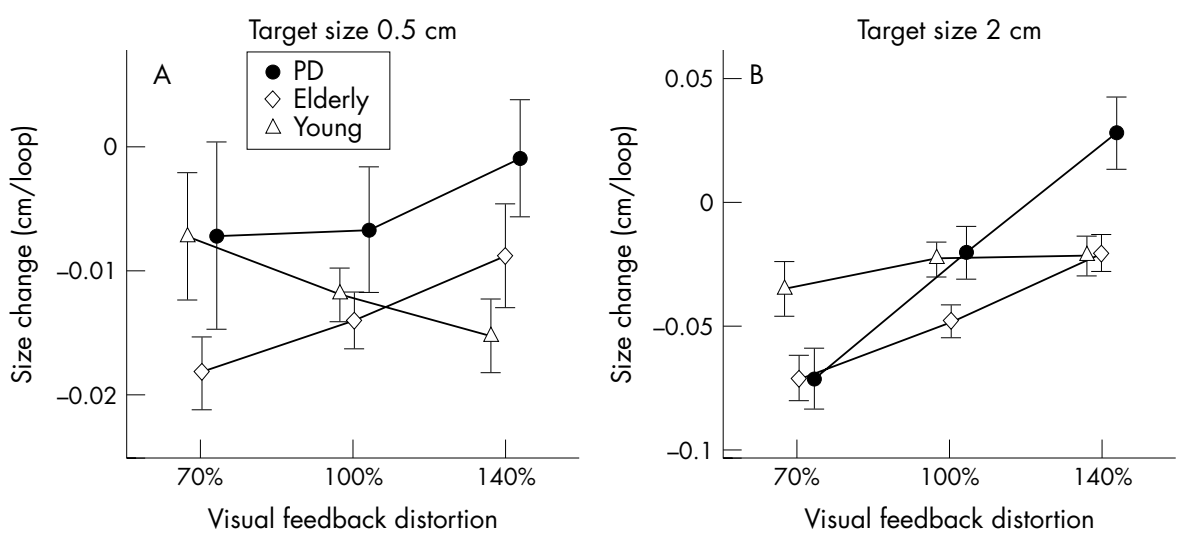

Figure 5 The size trend (expressed as the size change/loop across the first six loops/trial) for the $70 \%, 100 \%$, and $140 \%$ visual feedback conditions in the young controls, the elderly controls, and the patients with Parkinson's disease in (A) the $0.5 \mathrm{~cm}$ target size and (B) the $2 \mathrm{~cm}$ target size. The vertical bars are SEM. The patients with Parkinson's disease show, in the $2 \mathrm{~cm}$ target size, a large difference in trends between the $70 \%$ and the $140 \%$ conditions.

artificially create any of the results we reported. The trial by trial learning and adaptation of vertical stroke size in the $70 \%$ and the $140 \%$ conditions have, of course, opposite trends as supported by the condition by trial interaction $(F(10,25)=9.4$, $\mathrm{p}<0.001)$ in a three groups $\times$ two sizes $\times$ three conditions $\times$ six trials $\times$ six loops ANOVA comparing only the pre-exposure and the two exposure conditions (for example, fig 2 A and D). More importantly, the condition by trial interaction differs for the three groups as expressed by the group $\times$ condition $\times$ trial interaction $(F(20,50)=1.82, \mathrm{p}<0.05)$. We performed two sizes $\times$ six trials $\times$ six loops ANOVA for each group separately. Because the trial by trial adaptations in the $70 \%$ and the $140 \%$ conditions are in opposite directions and would cancel each other out, we performed these analyses within each distortion condition as well. This detailed analysis showed that the young controls displayed the expected trial by trial learning in both the $70 \%$ and in the $140 \%$ conditions $(F(5,5)=5.8$, $\mathrm{p}<0.05$, and $F(5,5)=6.9, \mathrm{p}<0.05$, respectively) (fig $2 \mathrm{~A}$ and $\mathrm{D}$ showing these effects in each target size). By contrast, the patients lacked any trial by trial adaptation effects in both the $70 \%$ and the $140 \%$ conditions $\left(F(5,6)=1.78, \mathrm{p}>0.2, \eta^{2}=0.60\right.$, power $=0.30$, and $F(5,6)=3.1, \mathrm{p}>0.1, \eta^{2}=0.72$, power $=0.49$, respectively) (fig $2 \mathrm{C}$ and F). Again, the elderly controls showed results in between those of the young controls and the patients (fig $2 \mathrm{~B}$ and $\mathrm{E}$ ).

\section{Loop by loop adaptation}

The loop by loop analysis provides information on the possible visuomotor mechanisms playing a part in adapting the vertical stroke size during handwriting. The young controls show the commonly found decrease of loop size during each handwriting trial (fig $4 \mathrm{~A}$ and $\mathrm{D}$ ). This is supported by the loop effect $(F(5,30)=13.9, \quad \mathrm{p}<0.001)$ in a three groups $\times$ two sizes $\times$ three conditions $\times$ six trials $\times$ six loops ANOVA, comparing only the pre-exposure and the $70 \%$ and the $140 \%$ conditions. The decrease of loop size has a linear trend across the first six loops $(F(1,34)=65, \mathrm{p}<0.001)$. This trend of loop size in the young controls (fig $5 \mathrm{~A}$ and $\mathrm{B}$ ) was almost identical in the $70 \%$, $100 \%$, and $140 \%$ visual feedback conditions-for example, in the $2 \mathrm{~cm}$ target size $-0.035,-0.024$, and $-0.022 \mathrm{~cm} / \mathrm{loop}$, respectively $(t(9)<1.0, \mathrm{p}>0.2$; sign test, $\mathrm{n}=10, \mathrm{k}>=3, \mathrm{p}>0.2)$. By contrast, the patients showed a remarkably different pattern (fig $4 \mathrm{C}$ and $\mathrm{F}$ ) as supported by the group by loop interaction $(F(10,60)=1.96, \mathrm{p}<0.05)$. Within each trial, the patients with Parkinson's disease progressively amplified the effect of the distortion as can be seen in the $2 \mathrm{~cm}$ target size. The patients showed significantly different loop size trends between the $70 \%, 100 \%$, and $140 \%$ visual feedback conditions $(-0.072,-0.021$, and $+0.027 \mathrm{~cm} / \mathrm{loop}$, respectively $(t(1))>3.6$, $\mathrm{p}<0.01$; sign test, $\mathrm{n}=12, \mathrm{k}<=1, \mathrm{p}<0.01$ ) (fig $5 \mathrm{~B}$ )). In the $140 \%$ condition, the patients with Parkinson's disease even showed a significant loop size increase compared with the elderly controls $(t(26)=3.2, \quad \mathrm{p}<0.01$; Mann-WhitneyWilcoxon $U(16,12)=174, \mathrm{p}<0.01)$. The elderly controls again showed results in between those of the young participants and the patients with Parkinson's disease (fig 4 B and E). In fact, in the elderly controls, loop size trends during each trial were slightly but significantly different between the $70 \%, 100 \%$, and $140 \%$ conditions $(-0.071,-0.049,-0.021 \mathrm{~cm} / \mathrm{loop}$, respectively $(t(15)>2.2, \mathrm{p}<0.05)$ (fig $5 \mathrm{~B}))$. Similar effects were seen in the $0.5 \mathrm{~cm}$ target sizes but they did not reach significance.

\section{DISCUSSION}

Patients with Parkinson's disease, elderly controls, and young adult controls wrote sequences of cursive l shaped loops while instructed to produce visible loop heights of $0.5 \mathrm{~cm}$ and $2 \mathrm{~cm}$ on a digitiser display. This digitiser enabled us to distort the visual feedback without the participant's knowledge by altering the gain of the vertical dimension of handwriting from $100 \%$ (real size) to $70 \%$ (reduced), then back to $100 \%$, then to $140 \%$ (enlarged) and finally back to $100 \%$ again. The results showed that the young adults gradually corrected loop size in the $70 \%$ distortion condition by enlarging (and widening) the size of the entire loop sequence and, conversely, in the 140\% distortion condition by reducing (and narrowing) the loop sizes so that the visible loop size was approximating the target size. The learning effects in the young adults were confirmed by the presence of significant after effects following each distortion condition and by the trial by trial improvements. Our results suggest that the young controls effectively used visual feedback to gradually update their visuomotor map in the size distorted feedback conditions. Apparently, they were able to detect a discrepancy of their original visuomotor map compared with the actual movement outcome and to adjust for the discrepancy, remember the most recent adjustment, and update the adjustment in a trial by trial learning process. Although the distortion did not affect the horizontal dimension, as this would cause highly noticeable cumulative discrepancies, the young controls adjusted also the horizontal component such that the ratio of horizontal and vertical motor amplitudes ("movement aspect ratio"), and not their visible aspect ratio, was maintained. This suggests that in the young controls, stroke direction is a higher order controlled variable than horizontal or vertical component size. ${ }^{30}$

The patients with Parkinson's disease, however, showed an entirely different response in comparison with the young controls: instead of correcting for the distortions, they progressively amplified the effect of the distortion of the vertical 
component. For example, in the $70 \%$ condition they progressively reduced their size and in the $140 \%$ condition they progressively enlarged their size. They altered also the horizontal component but they did that similarly to the young controls. Therefore, in the $70 \%$ condition, the handwriting of the patients became progressively smaller but wider (fig l B) and, in the $140 \%$ condition, progressively larger but narrower (fig 1 C). Moreover, the patients showed this effect in every trial, whereas there was no significant trial by trial learning and no after effects. This suggests that the patients with Parkinson's disease do not adapt their visuomotor map in response to the distorted visual feedback of handwriting. Instead, they seemed to rely constantly on the visible trace feedback during the ongoing movement. ${ }^{31-33}$ We hypothesise that they either plan their strokes on the basis of the visual feedback of their previous pen strokes or that they attempt to track the ongoing, distorted pen trace (matching action to perception). Both hypotheses are consistent with immediate and profound response of the patients with Parkinson's disease to distortions of the visual feedback. The hypothesised online visual feedback in the patients is supported by the finding that the effects are largely reduced in the $70 \%$ condition and in the 0.5 $\mathrm{cm}$ target size. Namely, in the $70 \%$ condition, the pen and the fingers occlude the direct visual feedback of the smaller than normal pen traces. Furthermore, the discrepancy between the actual movement and the distorted visual feedback is smaller in the $70 \%$ than in the $140 \%$ condition and especially, in the $0.5 \mathrm{~cm}$ condition $(-0.15 \mathrm{~cm} v+0.20 \mathrm{~cm}$, respectively, which is only a few times the display resolution of $0.03 \mathrm{~cm}$ ), and may be too small to trigger a response. The elderly controls showed results in between those of the young controls and the patients. The findings suggest that the elderly controls were not effectively using visual and proprioceptive feedback to update their visuomotor map as they showed reduced adaptation to the gain distortions. ${ }^{34}$

One of the first studies on the effects of visual feedback distortion on handwriting size in patients with Parkinson's disease and elderly controls was conducted by Fucetola and Smith. ${ }^{18}$ They used a paradigm where participants produced drawings on a normal digitiser and monitored their movements via a separate computer display, instead of a display digitiser as in our case. In the first block of trials, the visual feedback was normal $(100 \%)$. Then, in random order, two blocks of trials were performed where both the horizontal and vertical gain of the visual feedback were reduced to $50 \%$ or enlarged to $200 \%$, respectively. They found that the patients adjusted stroke size to roughly the same extent as the elderly controls and did not find any of the differences reported here. In a separate study to be reported elsewhere, we could replicate their results. That study was conducted with the same equipment and conditions as in our present experiment. The only difference was that visual feedback was not presented via the digitiser display but via a separate computer monitor instead. The five patients with Parkinson's disease, four age matched elderly controls, and four young controls were able to adapt equally well as evidenced by the absence of any group by condition interactions for any of the three stroke features $(F(8,40)<1)$.

The present results may help to elucidate possible hypotheses on the underlying causes of micrographia in Parkinson's disease. ${ }^{10}$ A hypothesis could be that patients with Parkinson's disease produce only slightly smaller strokes than they programmed-for example, due to reduced motor output or weakness of the agonist force ${ }^{35}$ - and fail to discover this discrepancy-for example, due to their reduced kinaesthesia ${ }^{3}$ - and programme the following strokes to match the visible stroke sizes (and not the intended sizes) of the previous, undersized strokes. Recursively repeating this process yields a progressive reduction of writing size as found in parkinsonian micrographia. Another hypothesis is that the patients with Parkinson's disease attempt to match their actions with their perception of the ongoing pen strokes. For example, in the $140 \%$ condition, when producing an upward stroke, the writing trace seems to run ahead of the pen tip while the patients use their visual feedback to speed up the pen towards the end point of the pen trace. During the downward stroke, the writing trace seems to lag behind the pen tip, causing the opposite effect. The second hypothesis was not entirely supported, as we found no significant duration effects between up and down strokes. Due to our necessarily unidimensional gain distortion we may not have seen the typical parkinsonian micrographia which is the reduction of both horizontal and vertical dimensions. ${ }^{10}$ Further research may be able to fine tune and discriminate between these and other underlying mechanisms of parkinsonian micrographia.

\section{ACKNOWLEDGEMENTS}

This research was supported by NINDS grant R01-NS-33173, the RS Flinn Foundation, and NIH SBIR grant R44-RR-11683.

\section{Authors' affiliations}

H-L Teulings, J L Contreras-Vidal, G E Stelmach, Motor Control Laboratory, Arizona State University, Tempe, Arizona 85287-0404, USA

C H Adler, Parkinson's Disease and Movement Disorder Center, Mayo Clinic, Scottsdale, AZ 85259, USA

\section{REFERENCES}

1 Marsden CD, Obeso JA. The function of the basal ganglia and the paradox of stereotaxic surgery in Parkinson's disease. Brain 1994; 117:877-97

2 Verschueren SM, Swinnen SP, Dom R, et al. Interlimb coordination in patients with Parkinson's disease: motor learning deficits and the importance of augmented information feedback. Exp Brain Res 1997; 1 13:497-508.

3 Demirci M, Grill S, McShane L, et al. A mismatch between kinesthetic and visual perception in Parkinson's disease. Ann Neurol 1997:41:781-8.

4 Jobst EE, Melnick ME, Byl NN, et al. Sensory perception in Parkinson's disease. Arch Neurol 1997;54:450-4.

5 Klockgether T, Borutta $M$, Rapp $H$, et al. A defect of kinesthesia in Parkinson's disease. Mov Disord 1995;10:460-5.

6 Klockgether T, Dichgans J. Visual control of arm movement in Parkinson's disease. Mov Disord 1994;9:48-56.

7 Rickards C, Cody FW. Proprioceptive control of wrist movements in Parkinson's disease: reduced muscle vibration-induced errors. Brain 1997; 120:977-90.

8 Schneider JS, Diamond SG, Markham CH. Parkinson's disease: sensory and motor problems in arms and hands. Neurology 1987;37:951-6.

9 Teulings HL, Stelmach GE. Control of stroke size, peak acceleration, and stroke duration in Parkinsonian handwriting. Human Movement Science 1991;10:315-33.

10 McLennan JE, Nakano K, Tyler HR, et al. Micrographia in Parkinson's disease. J Neurol Sci 1972;15:141-52.

11 Beuter A, Milton JG, Labrie C, et al. Delayed visual feedback and movement control in Parkinson's disease. Exp Neurol 1990;1 10:228_ 35.

12 Glickstein M. Stein J. Paradoxical movement in Parkinson's disease. Trends Neurosci 1991;14:480-2.

13 Diederich NJ, Goetz CG, Raman R, et al. Poor visual discrimination and visual hallucinations in Parkinson's disease. Clin Neuropharmacol 1998:21:289-95.

14 Poizner $\mathbf{H}$, Fookson Ol, Berkinblit $M B$, et al. Pointing to remembered targets in 3-D space in Parkinson's disease. Motor Control 1998;2:251-77

15 Smyth MM, Silvers G. Functions of vision in the control of handwriting Acta Psychologica 1987;3:276-315.

16 Vaillancourt DE, Slifkin AB, Newell KM. Intermittency in the visual control of force in Parkinson's disease. Exp Brain Res 2001;138:11827

17 Denier van der Gon JJ, Thuring JPh. The guiding of handwriting movements. Biol Cybern 1965;2:145-8.

18 Fucetola R, Smith MC. Distorted visual feedback effects on drawing in Parkinson's disease. Acta Psychologica 1997;95:255-66.

19 Van Gemmert AWA, Teulings HL, Stelmach GE. Parkinsonian patients reduce their stroke size with increased processing demands. Brain Cognition (in press).

20 Cools AR, Jaspers R, Schwarz M, et al. Basal ganglia and switching motor programs. In: McKenzie JS, Kemm RE, Wilcock LN, eds. The basa ganglia: structure and function. New York: Plenum Press, 1984:513-43.

21 Oliveira RM, Gurd JM, Nixon P, et al. Micrographia in Parkinson's disease: the effect of providing external cues. J Neurol Neurosurg Psychiatry 1997;63:629-32. 
22 Kagerer F, Contreras-Vidal JL, Stelmach GE. Adaptation to gradual versus sudden visuo-motor perturbations. Exp Brain Res 1997; 115:557-61

23 Shadmehr R, Mussa lvaldi MA. Adaptive representation of dynamics during learning of a motor task. J Neurosci 1994;14:3208-24.

24 Teulings HL, Maarse FJ. Digital recording and processing of handwriting movements. Human Movement Science 1984;3:193-217.

25 Burton AW, Pick HL, Holmes C, et al. The independence of horizontal and vertical dimensions in handwriting with and without vision. Acta Psychologica 1980;75:201-12.

26 Van Galen GP. Phonological and motoric demands in handwriting: evidence for discrete transmission of information. Acta Psychologica 1990;74:259-75.

27 Daniel WW. Applied nonparametric statistics. Boston: Houghton Mifflin, 1978.

28 Gibbons JD. Nonparametric methods for quantitative analysis. 2nd ed. Columbus, $\mathrm{OH}$ : American Sciences Press, 1985.
29 Maarse FJ, Thomassen AJWM. Produced and perceived writing slant: difference between up and down strokes. Acta Psychologica 1983;54:131-47.

30 Teulings HL, Schomaker LRB. Invariant properties between stroke features in handwriting. Acta Psychologica 1993;82:69-88.

31 Cooke JD, Brown JD, Brooks VB. Increased dependence on visua information for movement control in patients with Parkinson's disease. Can J Neurol Sci 1978:5:413-15.

32 Flash T, Inzelberg R, Schechtman E, et al. Kinematic analysis of upper limb trajectories in Parkinson's disease. Exp Neurol 1992;1 18:215-26.

33 Phillips JG, Stelmach GE. The contribution of movement disorder research to theories of motor control and learning. In: Summers J, ed. Approaches to the study of motor control and learning. Amsterdam: North-Holland, 1992:469-93.

34 Seidler-Dobrin RD, Stelmach GE. Persistence in visual feedback control by the elderly. Exp Brain Res 1998;119:467-74.

35 Margolin DI, Wing AM. Agraphia and micrographia: clinical manifestations of motor programming and performance disorders. Acta Psychologica 1983;54:263-83.

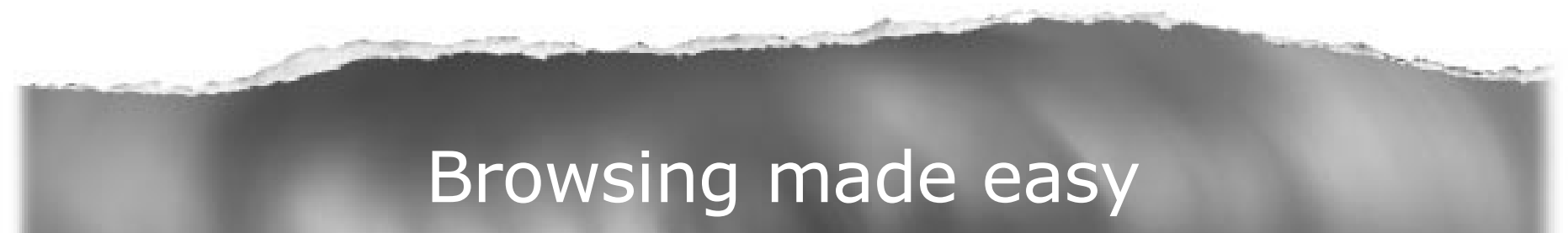

\section{Collections}

With a single click Collections allows you to find all articles that have been published in your chosen subject. Select from over 200 clinical and non-clinical topic collections and/or cross search other specialist journals, the BMJ and Cochrane Reviews

\section{www.jnnp.com}

